\title{
A NEW CONCEPT FOR REMOVAL OF DENTAL FLUOROSISS STAINS
}

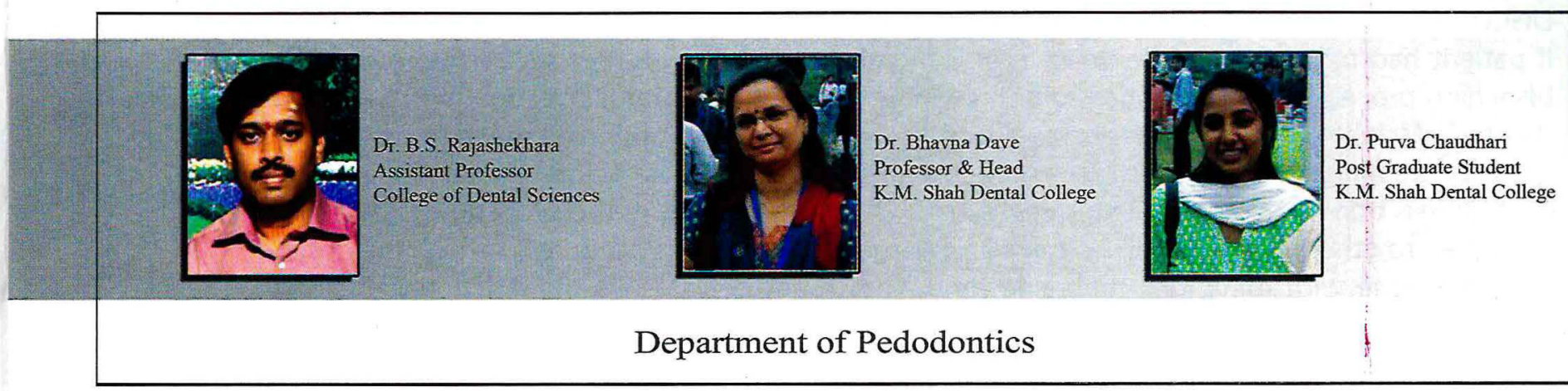

The objective of this case report was to evaluate the clinical efficacy of $5 \%$ sodium hypochlorite solution for removal of stains caused by dental fluorosis in young patients. The protocol of treatment 3 steps: (1) cleaning and enamel etching with $37 \%$ phosphoric acid in order to eliminate the layer that covers the fluorotic enamel surface and allow better penetration of the bleaching agent, (2) application of 5\% sodium hypochlorite to remove stains caused by organic material, and (3) filling the opened micro-cavities with a bonding agent to prevent restaining. The technique described in this case report appears to have advantages over other methods for improving the appearance of fluorotic lesions.

Key words - Dental Fluorosis, Sodium Hypochlorite, Phosphoric Acid.

\section{Introduction}

It is well documented that fluoride can have both beneficial and detrimental effects on the dentition ever since Mc Kay and G.V. Black in 1916 published the effect of fluoride on dentition'. It was Dean, 1934, who developed a classification for fluorosis, which is still widely used, based on his interpretation of clinical appearance ${ }^{2}$.Dean and McKay suggested that optimum level of water fluoride should be below $0.9-1.0 \mathrm{ppm}^{3}$. Human and animal studies have shown that the enamel hypo mineralization in fluorotic teeth are due to aberrant effects of fluoride on the rates at which enamel matrix protein breakdown or rates at which the byproducts of enamel matrix degradation are withdrawn, resulting in retardation of crystal growth in enamel maturation stage $\mathrm{e}^{4}$.
Significant numbers of patients visiting the pediatric dental clinics have esthetically objectionable brown stains and desire treatment for the same. Various treatment modalities are present for the treatment of fluorosis stains. Various treatment options available for dental fluorosis are micro abrasion, macro abrasion, micro abrasion with bleaching and laminates etc. This case reports discusses use of sodium hypochlorite for the improvement of dental esthetics in fluorosis.

\section{CASE REPORT}

A 12 year old male patient reported to the Pediatric Dentistry, K.M.Shah Dental College and Hospital, with a complaint of brown stains on the upper front teeth. Patient reported that these stains were present since the eruption of the teeth. His family had been living in the area of Vadodara for more than twenty years. He reported his siblings had the same condition. After the clinical examination a diagnosis of moderate grade of fluorosis according to Dean's Fluorosis index-Modified criteria was made (figure 1). The entire procedure was explained to the patient's parent and a written consent was obtained. Teeth were cleaned with flour of pumice using a rubber cup to remove all plaque \& rinsed with water .Proper isolation was done with the help of rubber dam application. The enamel surface was etched with $37 \%$ phosphoric acid for 15 seconds. The $5 \%$ Naocl was applied to the entire tooth surface using cotton applicator, repeating the application as the solution evaporated. The teeth were bleached for 15 to 20 minutes. To prevent organic material from reentering the porous \& hypomineralised enamel, the teeth was sealed with dentin bonding agent. After the procedure there was a complete elimination of the fluortic stains as can be seen in the post treatment photograph (figure 2). At two weeks follow up the patient reported no post operative sensitivity. 


\section{DISCUSSION}

If patient had mild grade of fluorosis in-office vital bleaching procedure with McInnes solution was advocated. Mclnnes solution has been successfully used for treating mild fluorosis. Advantage of this procedure is that it is relatively non-invasive

compared to other restorative procedures and also it could be done with minimum chair side time. The main disadvantage of this procedure is the postoperative sensitivity it produces and that it cannot be employed in patients with more severe grade of fluorosis5. Vital bleaching is more successful for

fluorosis in younger patients presenting with opaque to orange colour stain rather than older patients with darker type of brown stains ${ }^{6}$.

If patient had mild grade fluorosis and micro and macro abrasion were employed. Advantage of

micro and macro abrasion being its much faster procedure in achieving the desired result compared to other treatment options. However, the main

disadvantage is that these procedures employ high speed rotary instrument which can lead to excessive removal of tooth structure is operator does not have the desired skill level ${ }^{7}$.

Abrasion techniques can be successfully employed for discoloration presented either as single line discoloration or patchy type of discoloration, it cannot be successfully employed for discoloration which is more diffuse in nature8. Both the bleaching technique and abrasion procedures could be employed only for mild to moderate grade fluorosis. Most of the times, a combined treatment regimen of bleaching and abrasion procedures is employed to produce the desired aesthetic result in patients with yellowish discoloration due to fluorosis?

$5 \%$ sodium hypochlorite solution can be used for removal of stains caused by dental fluorosis in young patients10. Advantages of this procedure are simple, low cost, non invasive, relatively rapid \& safe, no special materials are required.

If the patient had moderate type of fluorosis which necessitated that the patient was treated by veneer procedure. Veneers have been successfully

employed for management moderate grade fluorosis ${ }^{11}$. Because of the time constraint given by patient, direct composite veneer treatment option was selected. Advantage of direct composite veneer is that it is done with minimal chair time when compared to indirect ceramic veneers, disadvantage being its long term wear resistance, color stability ${ }^{7}$.
Full mouth restorations were planned with metal ceramic crowns for a severe fluorosis patient requires careful investigations and preparation. This treatment option is limited to cases with severe fluorosis and loss of inter-occlusal space 12.

Advantage of this procedure is that it is an extensive procedure by which the desired aesthetic results and functional efficiency is achieved. The main disadvantage is also its extensiveness in treatment procedure which requires extensive lab procedure and operator skill, knowledge.

In each of the treatment options described above, each one has its own advantages and disadvantages; but the technique described in this case report appears to have advantages over other methods for improving the appearance of fluorotic lesions because it is simple, low cost, relatively rapid, safe and non invasive so the enamel keeps its structure, requires no special materials, and it can be used on young permanent teeth. A good clinician should be aware of all the treatment options available assess its merits and demerits and select the best treatment option according to individual patient needs.

\section{REFERENCES}

1) McKay FS, Black GV. Investigation of mottled teeth: An endemic imperfection of the enamel of teeth heretofore unknown in literature of dentistry. Dent Cosmos. 1916; 58:129.

2) Dean HT. Classification of mottled enamel diagnosis.J Am Dent Assoc.1934; 21:1421-6.

3) Dean HT, McKay FS. Production of mottled enamel halted by a change in common water supply. Am J Public Health. 1939;29:590-6.

4) Aoba T, Fejerskov O. Dental fluorosis: Chemistry and Biology. Crit Rev Oral Biol Med. 2002;13:155-70.

5) Greenwall L. An illustrated guide. New York: Martin Dunitz; 2001. Bleaching techniques in restorative dentistry; pp. 244-50.

6) Seale NS, Thrash WJ. Systematic assessment of colour removal following vital bleaching of intrinsically stained teeth.

J Dent Res. 1985;64:457-61. 
7) Roberson, Heymann, Swift Additional conservative esthetic procedures. 4th ed. Missouri: Mosby; 2002. Sturdevant's Art and Science of Operative dentistry; pp. 610-20.

8) Wong FS, Winter GB. Effectiveness of microabrasion technique for improvement of dental aesthetics. Br Dent J. 2002;193:155-8.

9) Sarrett DC. Tooth whitening today. J Am Dent Assoc. 2002;133:1535-8.[PubMed]

10) Aurora Cardenas Flores et al. Clinical efficacy of $5 \%$ sodium hypochlorite for removal of stains caused by dental fluorosis. JCPD 2009; 33(3):187-192.

11) Akapata ES. Occurrence and management of dental fluorosis. Int Dent J. 2001;51:325-33.

12) Ash, Ramfjord Occlusion in operative and restorative dentistry. 4th ed. Philadelphia: W B Saunders Company; Occlusion; pp. 409-22.

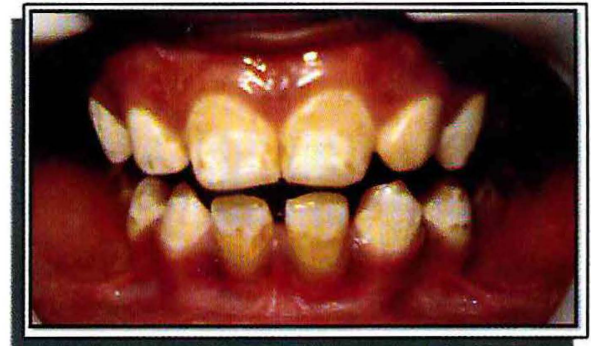

FIG 1: PRE-OPERATIVE

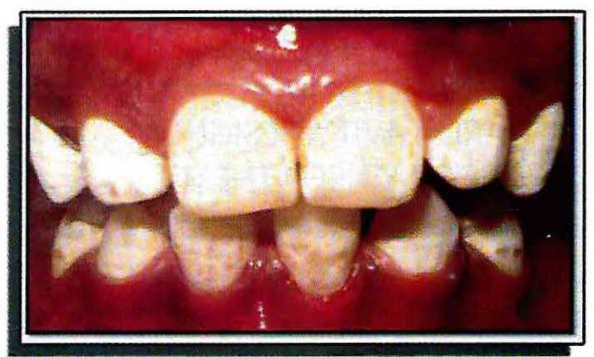

FIG 2: POST-OPERATIVE

\section{DEPARTMENTAL ACTIVITIES}

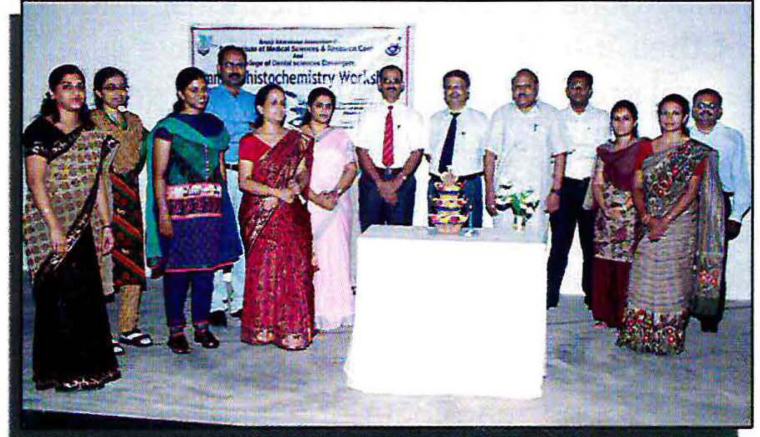

Immunohistochemistry workshop held on 26/07/2011 Organised by the Dept of Pathology

S S Institute of Medical Sciences \& Research centre and Dept of Oral Pathology College of ental Sciences, Davangere

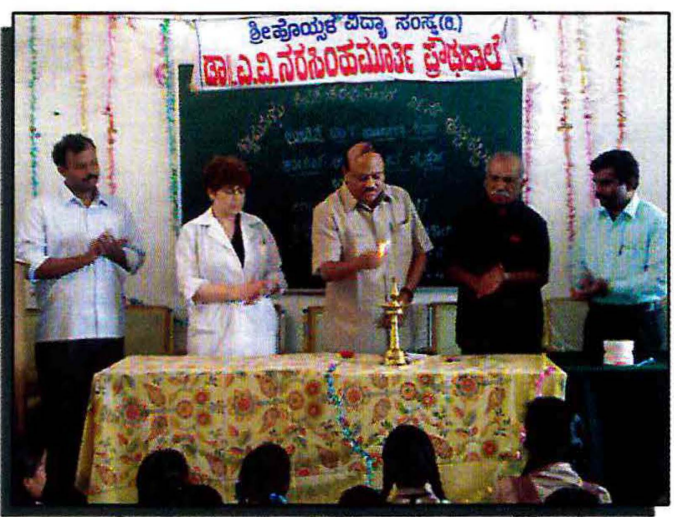

Free oral health screening and blood group identification camp held on occasion of 81st birthday of Sri Shamnur Shivashankrappa

Hon. Secretary BEA, Chairman, College of Dental sciences, Davangere conducted on 16-06-2011, in Dr. A V Narasimhamurthy High school, Lenin nagar Nituvalli , Davangere by the Dept of Oral Pathology and Dept of Community Dentistry, College of Dental Sciences, Davangere.

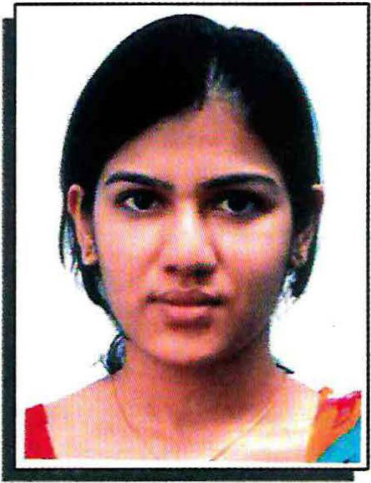

Dr. Bharati Maheshwari

III MDS Prosthodontics, Received Best paper presentation at 13th IPS National PG Student Convention at Babu Banarasi Das College of Dental Sciences, Lucknow on 24 to 26 June 2011. title of the paper "Full Mouth Rehabilitation of severely worn out dentition".

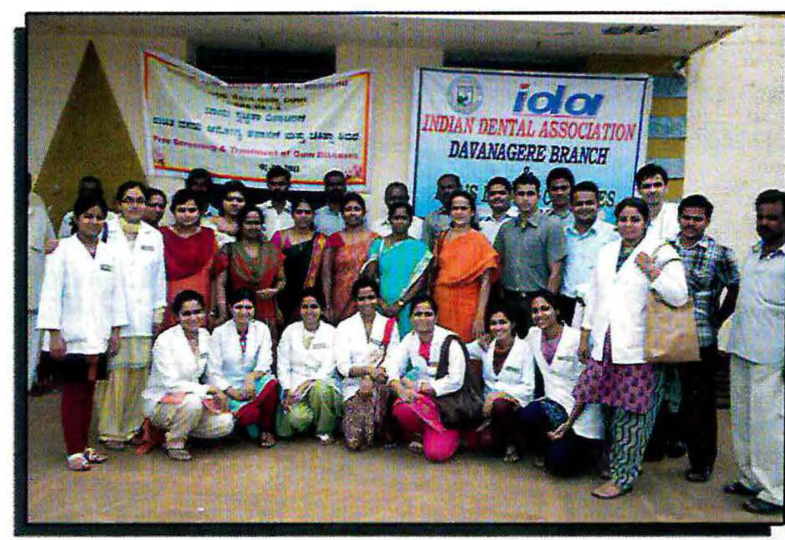

Oral Hygiene Camp conducted by Dept. of Periodontics at Koolambi village, Davangere held on 1/08/2011

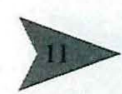

